\title{
WATER, METHANE, AND CARBON DIOXIDE PRESENT IN THE DAYSIDE SPECTRUM OF THE EXOPLANET
} HD 209458b

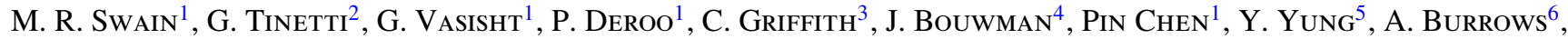 \\ L. R. Brown ${ }^{1}$, J. Matthews ${ }^{7}$, J. F. Rowe ${ }^{8}$, R. Kuschnig $^{9}$, And D. Angerhausen ${ }^{10,11}$ \\ ${ }^{1}$ Jet Propulsion Laboratory, California Institute of Technology, 4800 Oak Grove Drive, Pasadena, CA 91109, USA \\ ${ }^{2}$ University College London, Gower Street, London WC1E 6BT, UK \\ ${ }^{3}$ University of Arizona, Lunar and Planetary Laboratory, Space Science Building, Room 525, 1629 East, University Boulevard, Tucson, AZ 85721, USA \\ ${ }^{4}$ Max-Planck Institute for Astronomy, Konigstuhl 17, D-69117 Heidelberg, Germany \\ ${ }^{5}$ Division of Geological and Planetary Sciences, California Institute of Technology, Pasadena, CA 91125, USA \\ ${ }^{6}$ Department of Astrophysical Sciences, Princeton University, Princeton, NJ 08544, USA \\ ${ }^{7}$ Department of Physics \& Astronomy, University of British Columbia, Vancouver, BC V6T 171, Canada \\ ${ }^{8}$ NASA Ames Research Center, MS 244-30, Moffett Field, CA 94035, USA \\ ${ }^{9}$ Institut fur Astronomie, Universitat Wien Turkenschanzstrasse 17, A-1180 Wien, Austria \\ ${ }^{10}$ Institute of Space Systems, University Stuttgart, Pfaffenwaldring 31, 70569 Stuttgart, Germany \\ Received 2009 April 28; accepted 2009 August 21; published 2009 October 6
}

\begin{abstract}
Using the NICMOS instrument on the Hubble Space Telescope, we have measured the dayside spectrum of HD $209458 \mathrm{~b}$ between 1.5 and $2.5 \mu \mathrm{m}$. The emergent spectrum is dominated by features due to the presence of methane $\left(\mathrm{CH}_{4}\right)$ and water vapor $\left(\mathrm{H}_{2} \mathrm{O}\right)$, with smaller contributions from carbon dioxide $\left(\mathrm{CO}_{2}\right)$. Combining this near-infrared spectrum with existing mid-infrared measurements shows the existence of a temperature inversion and confirms the interpretation of previous photometry measurements. We find a family of plausible solutions for the molecular abundance and detailed temperature profile. Observationally resolving the ambiguity between abundance and temperature requires either (1) improved wavelength coverage or spectral resolution of the dayside emission spectrum or (2) a transmission spectrum where abundance determinations are less sensitive to the temperature structure.
\end{abstract}

Key words: planetary systems - techniques: spectroscopic

\section{INTRODUCTION}

HD 209458b has the distinction of being the first identified transiting extrasolar planet (Charbonneau et al. 2000). This 0.69 Jupiter-mass planet orbits a G0V stellar primary $\left(K_{\mathrm{mag}}=6.3\right)$ every 3.52 days and was the first exoplanet with a detected atmosphere (Charbonneau et al. 2002). It was also one of the first planets found to emit detectable amounts of infrared radiation (Deming et al. 2005). The dayside mid-infrared spectrum for this planet, based on Spitzer IRS observations, has been previously obtained (Richardson et al. 2007; Swain et al. 2008a). Midinfrared dayside photometry (Knutson et al. 2008) provides evidence for an atmospheric temperature inversion (Burrows et al. 2007) and has been used to support a classification scheme for the hot-Jupiter type exoplanets (Fortney et al. 2008). Midinfrared photometric measurements of the orbital phase light curve of HD 209458b (Cowan et al. 2007) find that the dayside and nightside brightness temperatures are similar. Models of atmospheric circulation for HD 209458b suggest that the zonal wind structure is a function of altitude (Showman et al. 2008), and mid-infrared dayside emission could vary (Rauscher et al. 2007, 2008; Showman et al. 2008).

The recent announcement of the detection of $\mathrm{H}_{2} \mathrm{O}$ and $\mathrm{CH}_{4}$ in the terminator regions of the hot-Jupiter HD 189733b (Swain et al. 2008b; hereafter SVT08) via near-infrared spectroscopy using the Hubble Space Telescope (HST) demonstrated that molecular spectroscopy of bright transiting planets is possible. The subsequent HST detection of $\mathrm{H}_{2} \mathrm{O}, \mathrm{CO}_{2}$, and carbon monoxide (CO) on the dayside of HD $189733 \mathrm{~b}$ provides significant constraints for atmospheric models and suggests that exoplanet molecular spectroscopy may become routine

\footnotetext{
${ }^{11}$ DAAD-Fellow
}

(Swain et al. 2009; hereafter S09) in the near future. This is significant because molecules provide a powerful tool for determining the conditions, composition, and chemistry of exoplanet atmospheres. In this paper, we report on the first ever spectroscopic detection of molecules in the atmosphere of HD 209458 b, making this the second exoplanet for which molecular spectroscopy has now been demonstrated.

\section{OBSERVATIONS AND DATA ANALYSIS}

We observed HD 209458 for five consecutive HST orbits using the NICMOS camera in imaging-spectroscopy mode covering the wavelengths $1.5-2.5 \mu \mathrm{m}$ using the G206 grism. As in previous observations (SVT08, S09), the DEFOCUS mode was used, resulting in a spectral resolution of $R \simeq 40$. Observations were conducted on June 15 2008, from UT 09:38:59 to UT 16:45:24. A total of 310 usable snapshot spectra were acquired with an individual exposure time of $T=4.06 \mathrm{~s}$. Using the system ephemeris (Knutson et al. 2007), we determine that the bulk of the first two orbits $\left(O_{1}\right.$ and $\left.O_{2}\right)$ coincides with the pre-ingress light curve, the third and part of the fourth orbit $\left(O_{3}\right.$ and $\left.O_{4}\right)$ cover the occultation, while the fifth orbit $\left(O_{5}\right)$ is post-egress (see Figure 1). We find that 75 exposures cover the full eclipse, 43 exposures are in ingress/egress, and 192 exposures cover the pre-ingress or post-egress intervals. The effective exposure time for each spectrum was $T=4.06 \mathrm{~s}$. To determine the spectrometer wavelength calibration, narrowband (F190) filter calibration exposures were acquired during $O_{1}$.

Our data analysis methods, based on decorrelation of instrument parameters from the measured spectrophotometric light curves, have been described previously (SVT08, S09). For the results presented here, the data-analysis approach remains largely unchanged but is updated in two significant ways. First, 


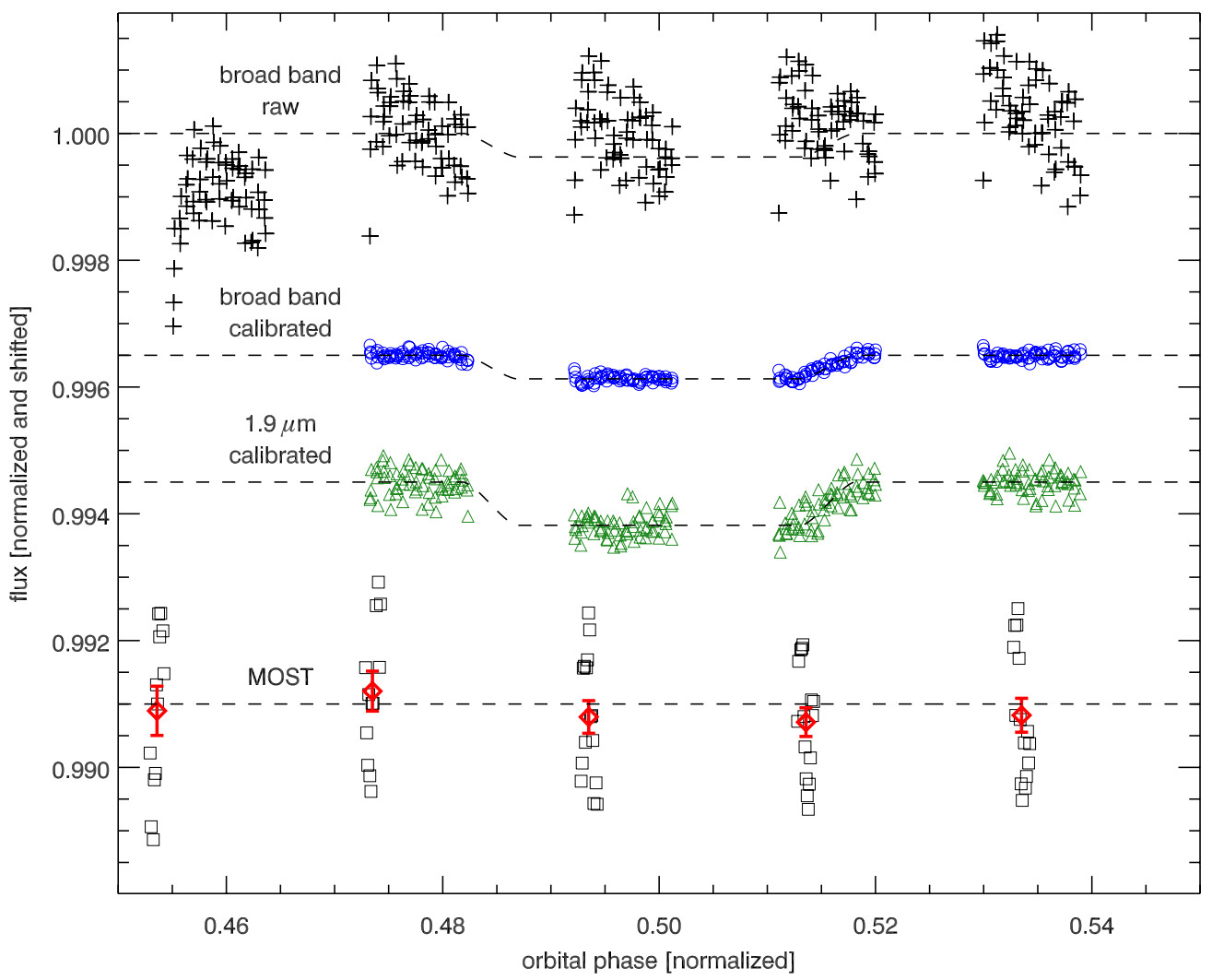

Figure 1. Set of normalized light curves plotted as a function of orbital phase. Gaps in the data are unavoidable because HD 209458 is not in the continuous viewing zone of Hubble. The top curve is the raw, broad-band, NICMOS data showing large internal scatter and a systematic offset affecting the first orbit. The second curve shows the calibrated, broad-band, NICMOS data (we exclude the first orbit); a shallow eclipse is visible. The third curve shows the calibrated $1.9 \mu$ m data. The larger eclipse depth at this wavelength (compared to the calibrated broadband data) is readily apparent. The fourth curve shows the contemporaneous MOST visible photometry data. Both the individual and averaged data are shown. The MOST data are consistent with no changes in the system brightness at visible wavelengths.

the decorrelation for instrument parameters is based on detector pixel values; in the past we averaged the detector data to the defocused spectral resolution prior to the decorrelation. The new approach has the advantage of determining the corrected, decorrelated spectrum using the full oversampling of the instrument. Second, we now derotate and shift the spectrum (via interpolation) to achieve the same sampling at all wavelengths and times. This effectively places observations in a common, pixel-based, reference frame. We tested this new reduction method by computing a dayside spectrum of HD 189733b and comparing it to our previous result (S09); the agreement between the two methods was found to be excellent, with differences between the spectra always within the statistical uncertainty of the measurement. As with our previous observations of the primary and secondary eclipse of HD 189733b, we found the instrument parameters for the first orbit, $O_{1}$, differed significantly from the remaining orbits, $\mathrm{O}_{2}-\mathrm{O}_{5}$; following our previous approach (SVT08, S09), we omitted the $O_{1}$ spectra from the analysis. To add an additional constraint to any possible orbit-to-orbit brightness changes, we arranged for contemporaneous, highprecision, visible photometry using the Micro-variability and Oscillations of Stars (MOST) satellite (Walker et al. 2003). The MOST observations were timed to provide a photometry measurement during each $H S T$ orbit (see Figure 1), and the data were calibrated using the method described by Rowe et al. (2008).

\section{DISCUSSION}

The near-infrared dayside spectrum of HD 209458b shows three clear peaks at $1.6,1.8-1.9$, and $2.2 \mu \mathrm{m}$, while between the peaks the flux density decreases to near-zero (within the measurement error). The spectrum has an average uncertainty per data point of $\sim 7.5 \times 10^{-5}$, corresponding to a dynamic range of 13,300:1 (75 ppm), with a wavelength resolution of $\frac{\lambda}{\Delta \lambda}=35$ at $2 \mu \mathrm{m}$. To maximize wavelength coverage for the spectral interpretation, we incorporated mid-infrared Spitzer photometry data (Knutson et al. 2008) and spectroscopy data (originally obtained by Richardson et al. 2007). We have previously undertaken our own calibration of these Spitzer IRS data (Swain et al. 2008a), and we use those results in the discussion that follows.

The interpretation of the data was done using two independent spectral retrieval methods incorporating a line-by-line radiative transfer model (Griffith et al. 1998; Tinetti et al. 2007a, 2007b, SVT08, S09), and the results from both models are in agreement. The radiative transfer calculations assume local thermal equilibrium (LTE) conditions, as expected for pressures exceeding $10^{-3}$ bar that are probed by the near-infrared spectrum. We evaluated a variety of temperature $(T)$ together with the effects of $\mathrm{CH}_{4}, \mathrm{H}_{2} \mathrm{O}$, and $\mathrm{CO}_{2}$, which are assumed to have constant mixing ratios (Figure 2). We use the $\mathrm{CH}_{4}$ line list of Nassar \& Bernath (2003) for temperatures at 800, 1000, and $1273 \mathrm{~K}$ to quantify the affects of $\mathrm{CH}_{4}$ at wavelengths of $1.58-2.5 \mu \mathrm{m}$, outside of which we use absorption parameters by Rotham et al. (2005). The hot water lines from Barber et al. (2006) and Zobov et al. (2008) were used to quantify the water features. Absorption by $\mathrm{CO}_{2}$ is calculated from the HITRAN hot $\mathrm{CO}_{2}$ line list (Tashkun et al. 2003). Absorption coefficients of $\mathrm{H}_{2} \mathrm{O}$ and $\mathrm{CO}_{2}$ are calculated using line-by-line techniques every $0.004 \mathrm{~cm}^{-1}$ wavenumbers. The radiative transfer calculation has 80 verti- 


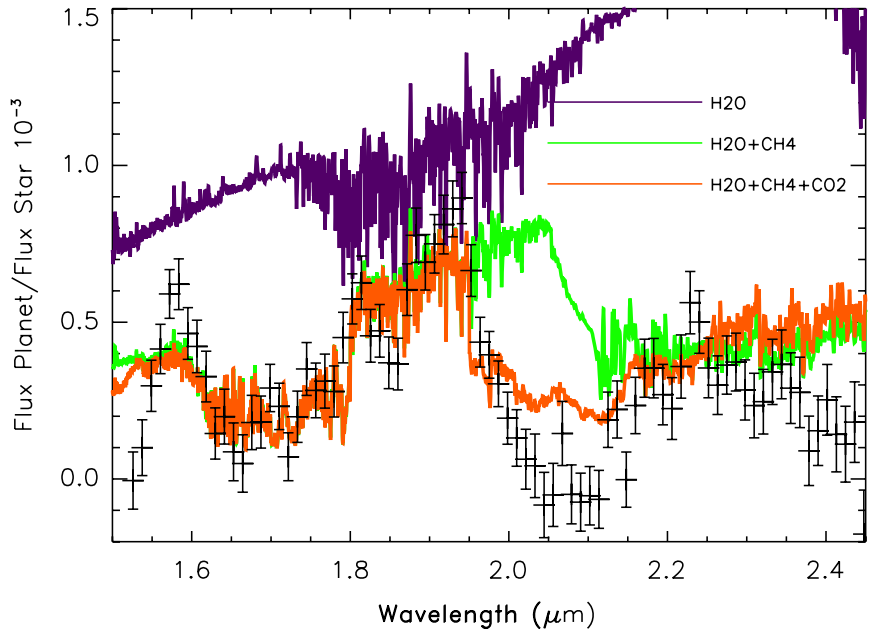

Figure 2. Comparison of the observed spectrum (black points with $\pm 1 \sigma$ error bars) to a sequence of models showing the affect of $\mathrm{H}_{2} \mathrm{O}, \mathrm{CH}_{4}$, and $\mathrm{CO}_{2}$. This comparison shows the portion of the near-infrared spectrum where each molecule makes a significant contribution. $\mathrm{CH}_{4}$ and $\mathrm{H}_{2} \mathrm{O}$ explain most of the spectral features, although additional absorption is needed around $2.0 \mu \mathrm{m}$ (provided by $\mathrm{CO}_{2}$ ). Here, the observed spectrum has been smoothed with a five-point scrolling boxcar and thus adjacent data points are not statistically independent.

cal grid points that extend from $10^{-7}$ to 10 bar. The effects of particulates are excluded.

The combination of near-infrared and mid-infrared data probes the temperature profile over a pressure range of a few bars to $10^{-5}$ bar and provides evidence for a temperature inversion. The $8 \mu \mathrm{m}$ brightness temperature is $1770 \mathrm{~K}$, while the 2.2-2.4 $\mu \mathrm{m}$ brightness temperature ranges from $1100-1600$ $\mathrm{K}$; this temperature difference, combined with the difference in pressure probed by these wavelengths, indicates the presence of a temperature inversion. We find that a temperature inversion could occur somewhere between $\sim 10^{-1}-10^{-4}$ bar, where temperatures increase by roughly $500-700 \mathrm{~K}$. This temperatureinverted region resembles those of planetary stratospheres; it occurs at a similar pressure level and causes a convectively stable temperature profile. The model spectrum from three exemplar temperature profiles (inverted) are shown in Figure 3 (together with a temperature profile with no inversion), and the model residuals (Figure 4) show the improved fit with a temperature inversion. The presence of the stratosphere coupled with a relatively steep stratospheric temperature gradient implies the existence of a local absorber that heats the atmosphere and maintains the local temperature structure. The temperature profile we derive confirms the previous identification of a $T$ inversion (Burrows et al. 2007; Knutson et al. 2008), but the profile reported here differs in terms of the detailed shape.

Our estimates for the temperature profile and molecular mixing ratios are based on modeling the combined nearinfrared and mid-infrared data sets. However, the recent finding of variability in the mid-infrared emission spectrum of HD 189733b (Grillmair et al. 2008) suggests caution is needed when deriving constraints from the noncontemporaneous midIR data. Although the presence of $\mathrm{H}_{2} \mathrm{O}$ and $\mathrm{CH}_{4}$ can explain most of the spectrum, we find that an additional absorber is required around $2.0 \mu \mathrm{m}$, and we attribute this to $\mathrm{CO}_{2}$. The evidence for a temperature inversion comes from the midinfrared spectrum, and the mid-infrared data provide some additional constraints on the mixing ratios for $\mathrm{H}_{2} \mathrm{O}$ and $\mathrm{CO}_{2}$. We find that a range of temperature profiles and molecular mixing ratios are consistent with the data as illustrated by the models shown in Figure 4 and the sample contribution functions shown in Figure 5, which correspond to three possible temperature profiles. Providing improved observational constraints on the location of the tropopause and the molecular mixing ratios will require additional observations, for which there are two
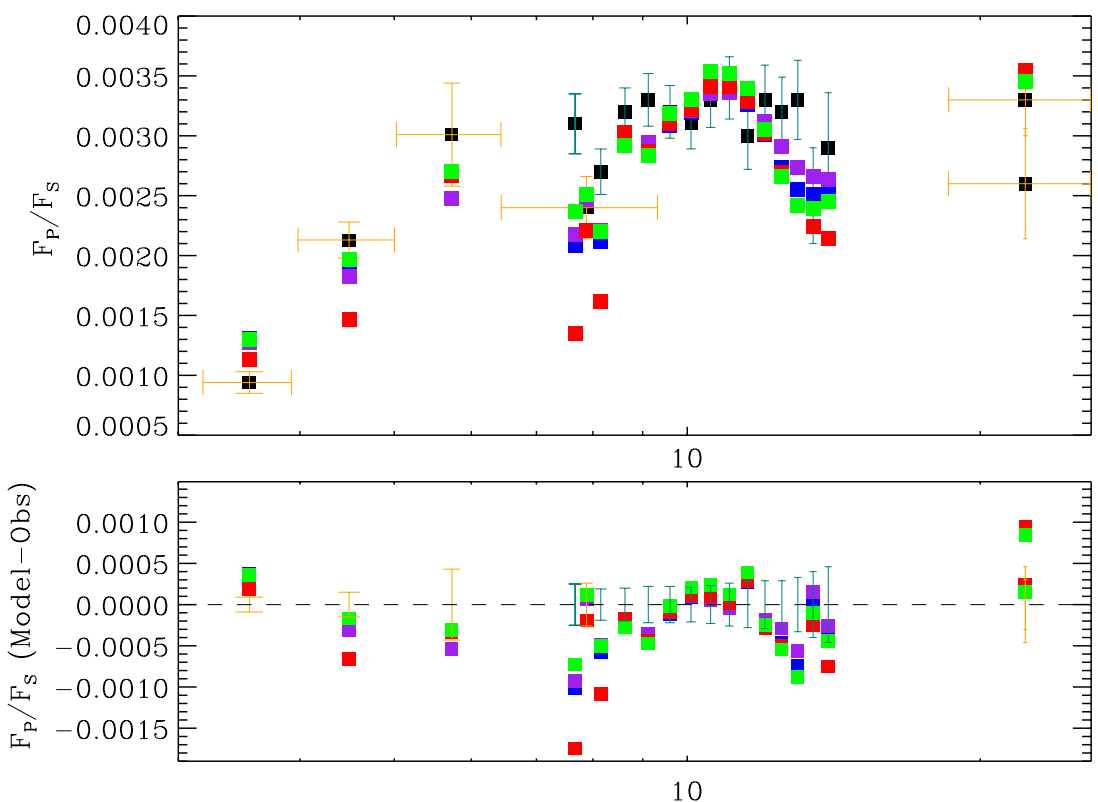

Wavelength $(\mu \mathrm{m})$

Figure 3. Top: mid-infrared observations compared to synthetic spectra for three models with the tropopause at 0.001 bars (blue), 0.01 bars (purple), and 0.1 bars (green); these models illustrate the range of temperature/composition possibilities consistent with the data. We also show the best-fitting model without a temperature inversion (red). Bottom: Model residuals compared to the mid-infrared observations. The model without a tropopause has the largest residuals. The contribution functions and temperature profiles associated with these models are shown in Figure 5. The mid-infrared photometry are from Knutson et al. (2008) and the mid-infrared spectroscopy are from Swain et al. (2008a). 


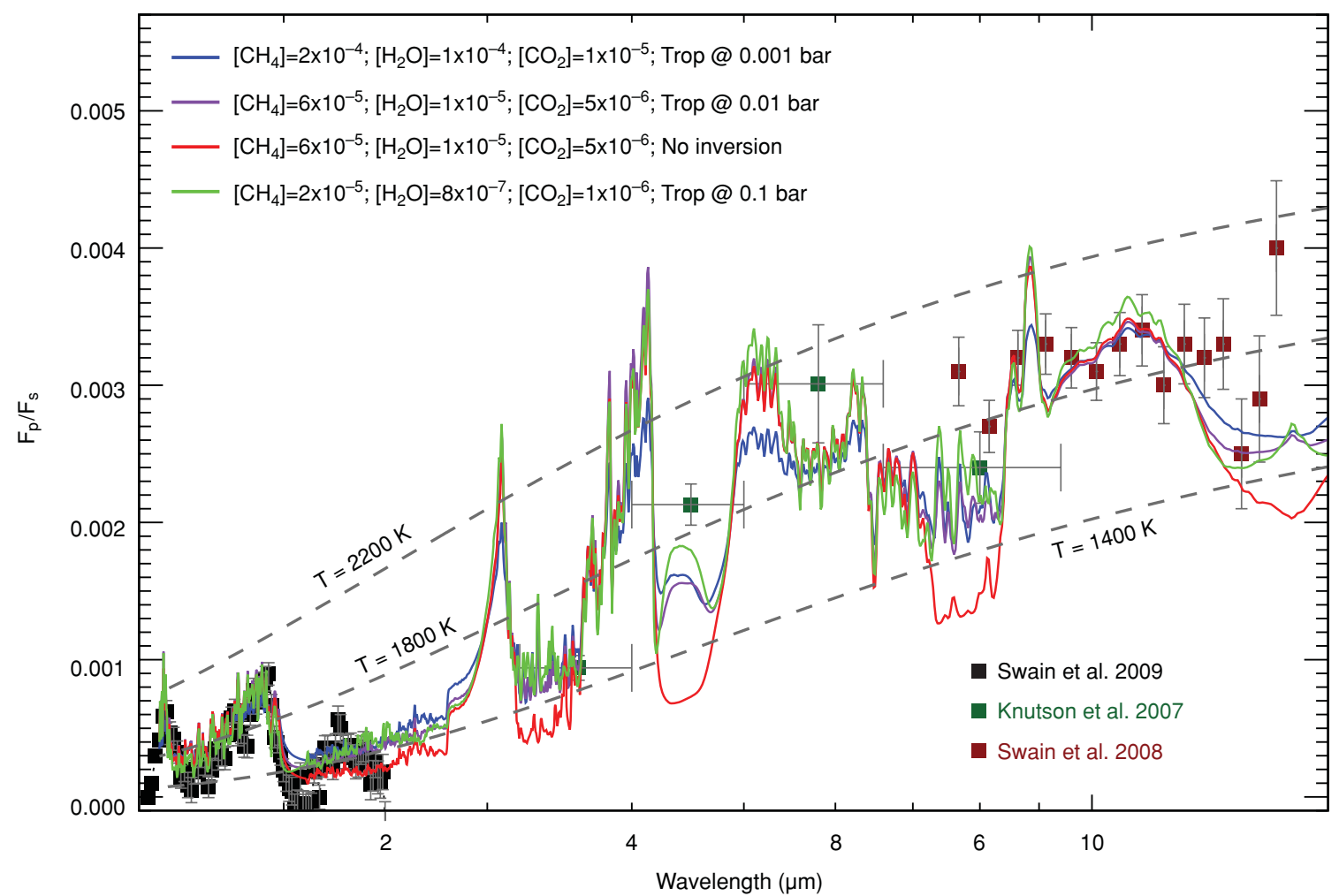

Figure 4. Near-infrared and mid-infrared observations compared to synthetic spectra for three models (also shown in Figure 3) that illustrate the range of temperature/ composition possibilities consistent with the data. For each model case, the molecular abundance of $\mathrm{CH}_{4}, \mathrm{H}_{2} \mathrm{O}$, and $\mathrm{CO}_{2}$ and the location of the tropopause is given; the contribution functions for each of these models is shown in Figure 5. The three dashed lines correspond to single-temperature thermal emission models with temperatures of 1400,1800 , and $2200 \mathrm{~K}$; these serve to illustrate how the combination of molecular opacities and the temperature structure cause significant departures from a purely single-temperature thermal emission spectrum. Note that the mid-infrared data are not contemporaneous with the near-infrared data, and attempting to "connect" these data sets with a model spectrum is potentially problematic if significant variability is present.
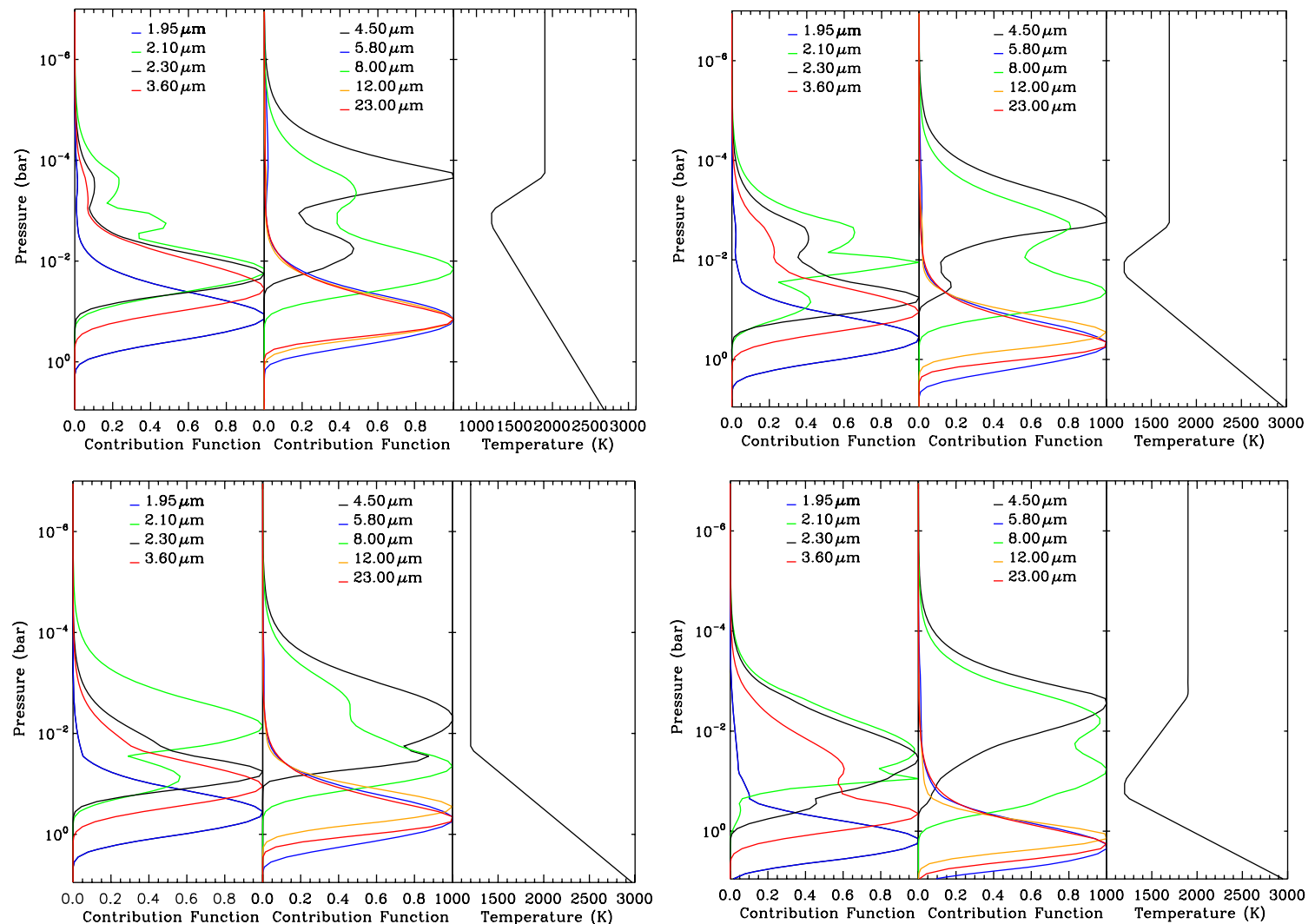

Figure 5. Contribution functions for selected wavelengths and the associated temperature profile for each model shown in Figures 3 and 4; starting from the top left and going clockwise, the models correspond to a tropopause at $0.001 \mathrm{bar}, 0.01 \mathrm{bar}, 0.1 \mathrm{bar}$, and no tropopause, respectively. The selected wavelengths correspond to bands of $\mathrm{H}_{2} \mathrm{O}$ and $\mathrm{CH}_{4}$; the contribution function is determined by opacity in each layer, which, in turn, can depend on the local temperature. 

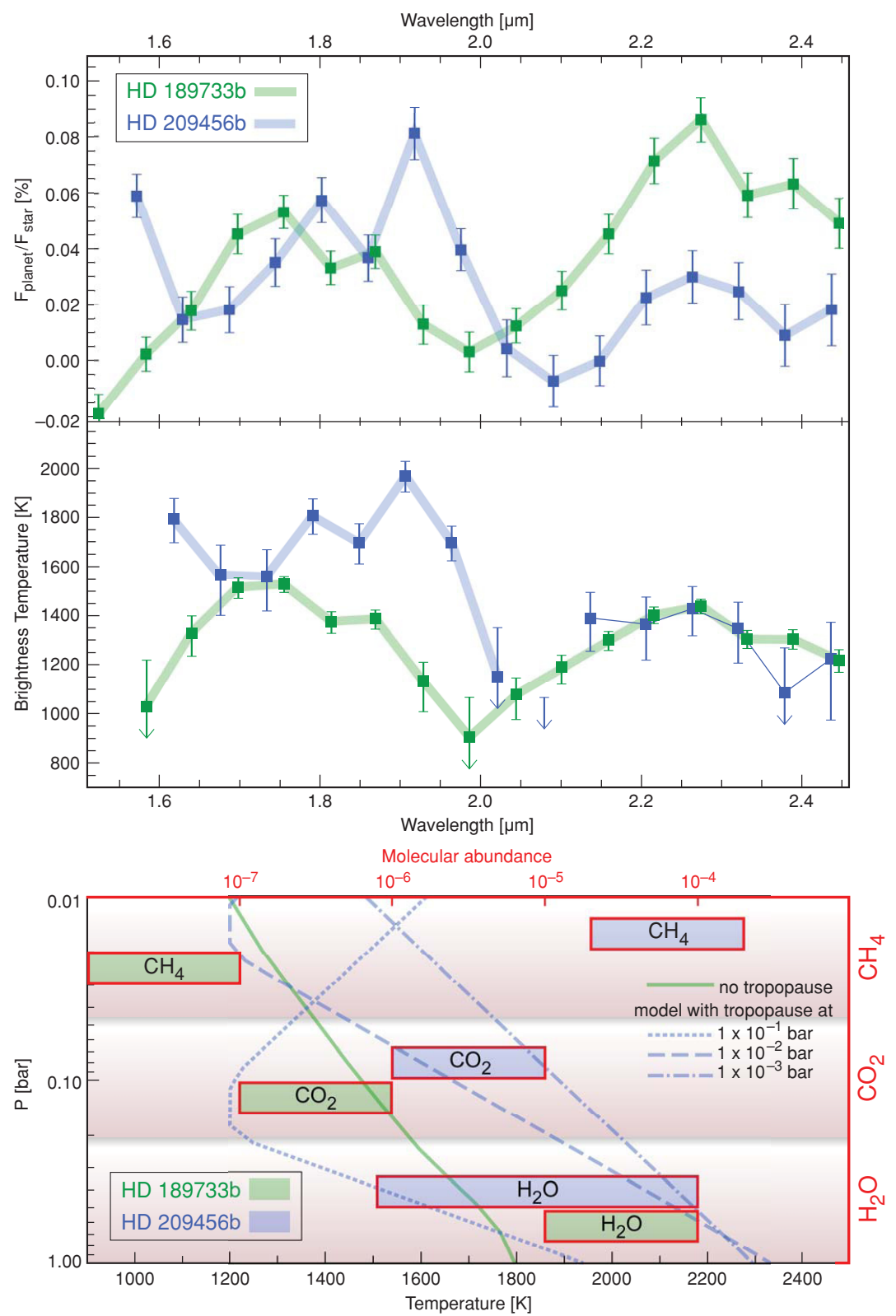

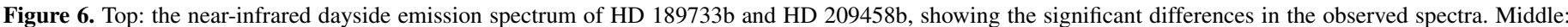

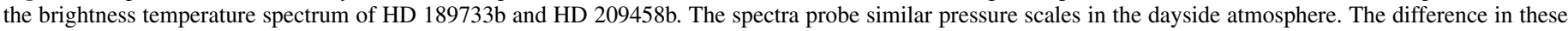

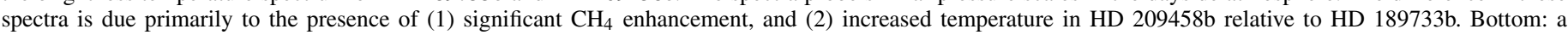

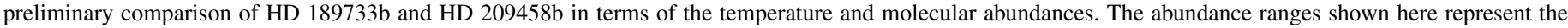

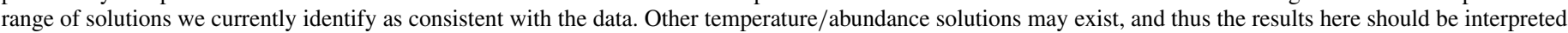

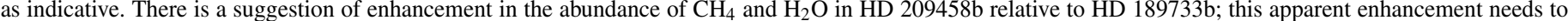
be confirmed via additional observations and detailed modeling.

possibilities. The first is to improve the spectral coverage and/ or spectral resolution of the dayside spectrum, which could be done in the future with James Webb Space Telescope (JWST). The second is to use a transmission spectrum (obtained during primary eclipse) to estimate the molecular abundances in the terminator regions; subject to some assumptions, this could be used to place constraints on the abundance of one or more molecules. HST transmission spectra for this planet have been obtained and are being analyzed by our team. However,the interpretation of the transmission spectrum is complex due to the presence of temporal variability (the subject of a forthcoming paper).

There are two important caveats concerning our derived temperature profiles. First is the assumption of emission from a uniform disk rather than from a realistic irradiated hemisphere; as such, the spectrum and T-profile we derive is disk averaged. Second, the spectral retrieval process used in our best-fitting models implicitly acknowledges the possible role of dynamics in establishing atmospheric temperature by relaxing typical one-line model constraints on the temperature gradient. Thus, portions of the atmosphere of HD 209458b may support departures from radiative equilibrium. Fully self-consistent modeling would require handling heat advection in the context of a global circulation model, which is beyond the scope of this paper. It is worth noting that the presence of a relatively strong dayside temperature inversion significantly complicates the spectral retrieval process for HD 209458 b relative to the case of HD 189733b. 
Given that the dayside emission spectra for HD $189733 \mathrm{~b}$ and HD 209458b have been observed with nearly identical instrument configurations, we can make a preliminary comparison of these two planets (see Figure 6). For the present, we restrict this discussion to the near-infrared to avoid the complications introduced by the presence of mid-infrared variability observed in HD 189733b (Grillmair et al. 2008). In both planets, the nearinfrared dayside emission spectrum probes the $5 \leqslant P \leqslant 10^{-2}$ bar portion of the atmosphere. Similarities - both planets show the presence of $\mathrm{H}_{2} \mathrm{O}, \mathrm{CO}_{2}$, and $\frac{d P}{d T} \leqslant 0$ for pressures near $1 \mathrm{bar}$. Differences - the abundance of $\mathrm{CH}_{4}$ is significantly enhanced in HD 209458b relative to HD 189733b, and the temperature at 1 bar is higher in HD 209458b, as well. The observed differences in the near-infrared dayside spectra of these two hot-Jupiters are likely due to differences in temperature and composition; HD209458b is dominated by $\mathrm{CH}_{4}$ absorption features, while $\mathrm{HD} 189733 \mathrm{~b}$ is dominated by absorption from $\mathrm{H}_{2} \mathrm{O}$ and $\mathrm{CO}_{2}$.

\section{CONCLUSIONS}

In summary, we have presented the first near-infrared spectrum of light emitted by HD 209458b. Using an iterative forward model approach for spectral retrieval, we find that $\mathrm{H}_{2} \mathrm{O}, \mathrm{CH}_{4}$, and $\mathrm{CO}_{2}$, together with a temperature inversion, are present in the dayside atmosphere of HD 209458b. There are a range of temperature profiles and molecular abundance solutions that are consistent with the data. Additional observational constraints on the atmospheric temperature structure and composition will require either improved wavelength coverage/spectral resolution for the dayside spectrum or a transmission spectrum. We note that some of the temperature profiles consistent for these observations raise the question of whether the dayside atmosphere is in radiative equilibrium. Although advection of heat could support departures from radiative equilibrium, our present knowledge of most molecular opacities at high temperatures limits our ability to determine decisively whether the radiative equilibrium condition is met or not; thus there is an urgent need for further laboratory studies to obtain molecular databases for determining high temperature opacities of the most common molecules expected in hot-Jupiter atmospheres.

We appreciate the Director's Time Award for these observations, and we thank Tommy Wiklind, Beth Padillo, and other members of the Space Telescope Science Institute staff for assistance in planning the observations. We also thank Jonathan Tennyson and Bob Barber for help with the water line list. G.T. was supported by the Royal Society. A portion of the research described in this paper was carried out at the Jet Propulsion Laboratory, under a contact with the National Aeronautics and Space Administration.

\section{REFERENCES}

Barber, R. J., Tennyson, J., Harris, G. J., \& Tolche nov, R. N. 2006, MNRAS, 368, 1087

Burrows, A., Hubeny, I., Budaj, J., Knutson, H. A., \& Charbonneau, D. 2007, ApJ, 668, 171

Charbonneau, D., Brown, T. M., Latham, D. W., \& Mayor, M. 2000, ApJ, 529, 45

Chabonneau, D., Brown, T. M., Noyes, R. W., \& Gilliland, R. L. 2002, ApJ, 568,377

Cowan, N. B., Agol, E., \& Charbonneau, D. 2007, MNRAS, 379, 641

Deming, D., Seager, S., Richardson, L. J., \& Harrington, J. 2005, Nature, 434, 740

Fortney, J. J., Marley, M. S., Saumon, D., \& Lodders, K. 2008, ApJ, 683, 1104

Griffith, C. A., Yelle, R. V., \& Marley, M. S. 1998, Science, 282, 2063

Grillmair, C. J, et al. 2008, Nature, 456, 767

Knutson, H. A., Charbonneau, D., Allen, L. E., Burrows, A., \& Thomas, M. S. 2008, ApJ, 673, 526

Knutson, H. A., Charbonneau, D., Noyes, R. W., Brown, T. M., \& Gilliland, R. L. 2007, ApJ, 655, 564

Nassar, R., \& Bernath, P. 2003, J. Quant. Spectrosc. Radiat. Transfer, 82, 279

Rauscher, E., Kristen, M., Cho, J. Y.-K, Seager, S., \& Hansen, B. M. S. 2008, ApJ, 681, 1646

Rauscher, E., et al. 2007, ApJ, 664, 1199

Rotham, L. S., et al. 2005, J. Quant. Spectrosc. Radiat. Transfer, 92, 139

Rowe, J. F. 2008, ApJ, 689, 1345

Richardson, J. L., Deming, D., Horning, K., Seager, S., \& Harrington, J. 2007, Nature, 445, 892

Showman, A. P., Cooper, C. S., Fortney, J. J., \& Marley, M. S. 2008, ApJ, 682, $559 \mathrm{~S}$

Swain, M. R., Bouwman, J., Akeson, R. L., Lawler, S., \& Beichman, C. A. 2008a, ApJ, 674, 482

Swain, M. R., Vasisht, G. V., \& Tinetti, G. 2008b, Nature, 452, 329

Swain, M. R., et al. 2009, ApJ, 960, L114

Tashkun, S. A., Perevalov, V. I., Tefflo, J.-L., Bykov, A. D., \& Lavrentieva, N. N. 2003, J. Quant. Spectrosc. Radiat. Transfer, 82, 165

Tinetti, G., et al. 2007a, ApJ, L99

Tinetti, G., et al. 2007b, Nature, 448, 169

Walker, G., et al. 2003, PASP, 115, 1023

Zobov, N. F., et al. 2008, MNRAS, 387, 1093 